\title{
Aplicação dos isótopos estáveis em aqüicultura
}

\section{Carlos Ducatti}

UNESP-Instituto de Biociências. Centro de Isótopos Estáveis. Distrito de Rubião Júnior, S/N. Botucatu, SP. CEP 18.618-000. ducatti@ibb.unesp.br

RESUMO - O produto mais importante do conhecimento é a ignorância. A situação sugere a imagem de alguém que vive numa ilha de conhecimento cercado por um mar de ignorância. Quando a área dessa ilha aumenta pelo progresso da ciência, também aumenta o litoral, - a fronteira com o desconhecido. Esta expansão é o fruto mais valioso do conhecimento (David Gross, Nobel em Física, 2004).

O uso dos isótopos estáveis dos bioelementos (CHON'S) presentes na Atmosfera, Hidrosfera, Litosfera e Biosfera enquadra-se neste contexto, surgindo como ferramenta de alto potencial em pesquisas nas diversas áreas das Ciências da Vida.

Com organismos aquáticos, a aplicação dos isótopos estáveis do carbono $\left(\delta^{13} \mathrm{C}\right)$ e do nitrogênio $\left(\delta{ }^{15} \mathrm{~N}\right)$ tem ocorrido em diversas áreas. Em larvas de pacu Piaractus mesopotamicus, a técnica evidenciou a escolha voluntária entre organismos vivos e dieta seca pelos animais, desde as primeiras alimentações até o final da larvicultura. Na carcinicultura, o crescimento do Penaeus vannamei resultou do aproveitamento dos alimentos oferecidos e da produtividade primária do lago (biota). Por meio do balanço de massa e isotópico foi possível determinar a contribuição da biota, da ordem de 44 a $86 \%$ do carbono assimilado pelos animais durante o crescimento. Outro exemplo é com o caranguejo de mangue Sesarma rectum, em que sua preferência alimentar por Spartina alterniflora $\left(\mathrm{C}_{4}\right)$ versus Rhizophora mangle $\left(\mathrm{C}_{3}\right)$ ou Hibiscus tiliaceus $\left(\mathrm{C}_{3}\right)$ foi evidenciada pelo modelo isotópico de duas fontes alimentares e os isótopos estáveis do carbono $\left(\delta^{13} \mathrm{C}\right)$.

A técnica dos isótopos estáveis associada ao conhecimento dos conceitos básicos da variabilidade isotópica natural e ao uso criterioso das razões ${ }^{13} \mathrm{C} /{ }^{12} \mathrm{C} ;{ }^{2} \mathrm{H} /{ }^{1} \mathrm{H} ;{ }^{18} \mathrm{O} /{ }^{16} \mathrm{O} ;{ }^{15} \mathrm{~N} /{ }^{14} \mathrm{~N}$ e ${ }^{36} \mathrm{~S} /{ }^{34} \mathrm{~S}$ revela-se como um avanço na fronteira dos conhecimentos, nas diversas reentrâncias do litoral da aqüicultura.

Palavras-chave: aqüicultura, carbono-13, isótopos estáveis, nitrogênio-15

\section{Stable Isotopes in Aquaculture}

SUMMARY - "The most important collateral effect of knowledge is ignorance. Just imagine someone else living in the knowledge island circulated by a sea of ignorance. When the surface of the island grows through the progress of science, also grows its border, its perimeter length, the frontier with the unknown". (David Gross, 2004 Nobel laureate)

The use of stable isotopes of bioelements (CHONS) are present in atmosphere, hydrosphere, lithosphere and biosphere, emerging as high potential tools for researches within different areas of Life Science. With aquatic organisms, the application of stable isotope of carbon $\left(\delta^{13} \mathrm{C}\right)$ and of nitrogen $\left(\delta^{15} \mathrm{~N}\right)$ has occurred in different areas. In pacu Piaractus mesopotamicus larvae, the technique showed the free choice of the animals between live organisms and dry diet, during the whole larvae life since the first feeding. In shrimp culture, the growth of Paneus vannamei resulted from the metabolism of food provided and the lake primary productivity (biota). Through the mass and isotopic balance it was possible to measure the biota contribution, around $44 \%$ to $86 \%$ of carbon assimilated by the animals during its growth. Another example is related with the mangue crab Sesarma rectum, whose preference nutrition among Spartina alterniflora $(\mathrm{C} 4)$, Rhizophora mangle (C3) and Hibiscus tiliaceus (C3) was evidenced by the isotopic model of two food sources using stable isotope of carbon $\left({ }^{13} \mathrm{C}\right)$. The technique of stable isotopes associated with the knowledge of basic concepts of natural isotopic variability and with the careful use of ratios ${ }^{13} \mathrm{C} /{ }^{12} \mathrm{C},{ }^{2} \mathrm{H} /{ }^{1} \mathrm{H},{ }^{18} \mathrm{O} /{ }^{16} \mathrm{O},{ }^{15} \mathrm{~N} /{ }^{14} \mathrm{~N}$ and ${ }^{36} \mathrm{~S} /{ }^{34} \mathrm{~S}$ reveals itself as an advance for the knowledge island, in aquaculture area.

Key Works: Aquaculture, carbon-13, nitrogen-15, stable isotopes 


\section{Os isótopos estáveis e sua ocorrência na natureza}

Os isótopos estáveis do carbono (C), hidrogênio $(\mathrm{H})$, oxigênio $(\mathrm{O})$ e nitrogênio $(\mathrm{N})$ ocorrem naturalmente na atmosfera, hidrosfera, litosfera e biosfera. O termo isótopo vem do grego ISO (mesmo ou igual) e TOPOS (lugar), referindo-se ao fato de que ocupam o mesmo lugar na tabela periódica. A expressão estáveis significa que não emitem radiação. Os isótopos são átomos do mesmo elemento químico (mesmo número de prótons), mas que diferem em número de nêutrons, apresentando diferentes massas. Cada elemento químico apresenta um isótopo estável leve, dominante: carbono-12 $\left({ }^{12} \mathrm{C}\right)$, hidrogênio-1 $\left({ }^{1} \mathrm{H}\right)$, oxigênio-16 $\left({ }^{16} \mathrm{O}\right)$ e nitrogênio-14 $\left({ }^{14} \mathrm{~N}\right)$, e um ou dois isótopos pesados: carbono-13 $\left({ }^{13} \mathrm{C}\right)$, hidrogênio-2 $\left({ }^{2} \mathrm{H}\right)$, oxigênio-17 $\left({ }^{17} \mathrm{O}\right)$, oxigênio-18 $\left({ }^{18} \mathrm{O}\right)$ e nitrogênio-15 $\left({ }^{15} \mathrm{~N}\right)$, com abundância ou concentração natural expressa em átomos \% (Tabela 1).

Os valores do enriquecimento relativo do carbono-13 são expressos na terminologia dos isótopos estáveis, em delta per mil (\%o) da razão isotópica ${ }^{13} \mathrm{C} /{ }^{12} \mathrm{C}$ da amostra em relação ao padrão internacional, PeeDee Belemnite (PDB).

Para os isótopos estáveis do nitrogênio, o padrão internacional aceito é o do ar atmosférico, o qual é considerado uma mistura isotópica homogênea na superfície terrestre.

A terminologia empregada no estudo da variabilidade isotópica dos elementos químicos é resultante da expressão (1):

$\delta \mathbf{x}$ $=[(\mathbf{R}$ $\left.\left.{ }_{\text {nostra }} / \mathbf{R}_{\text {padrão }}\right)-1\right] \cdot 10^{3}$

A simbologia adimensional empregada na equação (1) significa: $\delta x_{\text {(amostra, padrão) }}$ é o enriquecimento da razão isotópica do elemento químico em questão, de uma dada amostra em relação ao seu respectivo padrão internacional, em partes per mil (\%o) e R significa razão isotópica do isótopo pesado em relação ao leve $\left(\mathrm{ex}:{ }^{13} \mathrm{C} /{ }^{12} \mathrm{C}\right.$; ${ }^{15} \mathrm{~N} /{ }^{14} \mathrm{~N}$ ) da amostra e do padrão, respectivamente.

As mensurações dos valores isotópicos $\delta^{13} \mathrm{Ce}$ $\delta^{15} \mathrm{~N}$ são efetuadas no espectrômetro de massa de razões isotópicas (IRMS), com erro analítico da ordem de $0,2 \%$. Valores isotópicos negativos significam que a amostra apresenta, relativamente, menos isótopo pesado que o padrão internacional.

A fonte primária do carbono para as plantas terrestres é o $\mathrm{CO}_{2}$ atmosférico, o qual possui valor de $\delta^{13} \mathrm{C}$ de aproximadamente $-7,7 \%$. De acordo com seu ciclo fotossintético, as plantas podem ser classificadas, na sua maioria, em dois grupos principais: $\mathrm{C}_{3}$ e $\mathrm{C}_{4}$, onde o primeiro composto orgânico a ser sintetizado é o ácido carboxílico, com três e quatro átomos de carbono, respectivamente. A via fotossintética $\mathrm{C}_{3}$ inclui a maioria das árvores, arbustos, soja, arroz, trigo, cevada entre outros, e a via $\mathrm{C}_{4}$ é encontrada no milho, sorgo, cana-de-açúcar e outras gramíneas tropicais.

Estas informações são ilustradas na Figura 1.

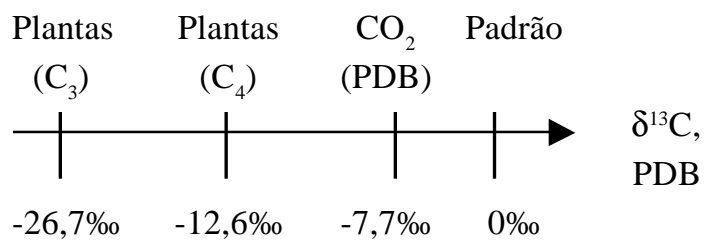

Figura 1 - Régua isotópica com escala em $\delta^{13} \mathrm{C}$, PDB.

Essas diferenças naturais no enriquecimento relativo do carbono $\left(\delta^{13} \mathrm{C}\right)$ entre espécies de plantas oferecem a possibilidade de utilizar essas plantas como traçadores em animais, sem a necessidade de sintetizar compostos especificamente marcados, além de evitar os problemas de saúde e segurança associados ao uso de isótopos radioativos.

As investigações científicas demonstraram que as composições isotópicas dos tecidos de animais dependem principalmente da alimentação, da água

Tabela 1 - Abundância natural dos isótopos estáveis em átomos \%.

\begin{tabular}{ccccc}
\hline \multicolumn{2}{c}{ Isótopo leve } & \multicolumn{2}{c}{ Isótopo pesado } & Padrão internacional \\
\hline${ }^{12} \mathrm{C}$ & 98,892 & ${ }^{13} \mathrm{C}$ & 1,108 & V-PDB \\
${ }^{1} \mathrm{H}$ & 99,985 & ${ }^{2} \mathrm{H}$ & 0,015 & V-SMOW \\
${ }^{16} \mathrm{O}$ & 99,759 & ${ }^{17} \mathrm{O}$ & 0,0374 & V-SMOW e PDB \\
& & ${ }^{18} \mathrm{O}$ & 0,2036 & \\
${ }^{14} \mathrm{~N}$ & 99,6337 & ${ }^{15} \mathrm{~N}$ & 0,3663 & $\mathrm{~N}_{2}$ atm \\
${ }^{32} \mathrm{~S}$ & 95,02 & ${ }^{34} \mathrm{~S}$ & 4,22 & V-CDT \\
\hline
\end{tabular}

๑) 2007 Sociedade Brasileira de Zootecnia 
ingerida e dos gases inalados, assim como, dos processos metabólicos (Kennedy \& Krouse, 1990).

\section{Turnover Isotópico}

Cada tecido ou fração bioquímica pode apresentar memória isotópica própria em função do conteúdo isotópico da alimentação e da taxa de renovação bioquímica.

Entende-se turnover isotópico como sendo a renovação contínua dos elementos químicos e, conseqüentemente, de seus isótopos que compõem o tecido corporal ou o organismo como um todo. O turnover pode ocorrer por meio de renovação tecidual resultante do processo de síntese e degradação em tecidos adultos e/ou pelo próprio crescimento nos tecidos em formação (diluição isotópica).

Ducatti et al. (2002), fundamentados em resultados da literatura, propuseram um modelo teórico-experimental capaz de exprimir os resultados do enriquecimento relativo de carbono $\left(\delta^{13} \mathrm{C}\right)$ em função do tempo de alimentação, fornecendo dietas contrastantes em $\delta^{13} \mathrm{C}$ (formuladas com grãos de plantas dos ciclos fotossintéticos $\mathrm{C}_{3}$ e $\mathrm{C}_{4}$ ) à animais adultos. Partindo do pressuposto que $\mathrm{o}$ metabolismo de determinado tecido animal depende da taxa de entrada de compostos de carbono da dieta e da taxa de substituição dos compostos préexistentes, os autores propõem o uso da equação (2):

$$
\delta^{13} \mathrm{C}_{(\mathrm{t})}=\delta^{13} \mathrm{C}_{(\mathrm{f})}+\left[\delta^{13} \mathrm{C}_{(\mathrm{i})}-\delta^{13} \mathrm{C}_{(\mathrm{f})}\right] \mathrm{e}^{-\mathrm{kt}}
$$

Na expressão (2), a simbologia adimensional empregada significa:

$\boldsymbol{\delta}^{\mathbf{1 3}} \mathbf{C}_{(\mathbf{t})}$ : enriquecimento isotópico do carbono no tempo desejado;

$\boldsymbol{\delta}^{13} \mathbf{C}(\boldsymbol{i})$ : enriquecimento isotópico inicial no tecido;

$\boldsymbol{\delta}^{3} \mathbf{C}_{(f)}$ : enriquecimento isotópico do carbono final; $k$ é a constante de troca de carbono e $t$ é a unidade de tempo.

O modelo é adequado para determinar o tempo de substituição total ou parcial (meia-vida, $\mathrm{T}=\ln$ $2 / k$ ) de carbono nos tecidos em estudos com aves e animais de pequeno porte. Estes conceitos são ilustrados na Figura 2.

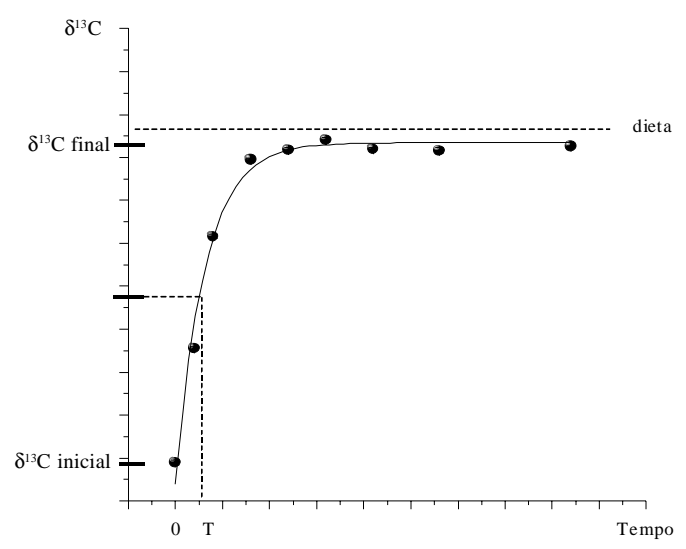

Figura 2 - Turnover isotópico e meia-vida (T).

Análises da variação natural da abundância de isótopos estáveis apresentam-se como uma ferramenta potencialmente poderosa para as pesquisas em fisiologia, uma vez que a razão isotópica dos elementos do corpo dos consumidores geralmente assemelha-se a suas dietas.

O período sobre o qual a concentração isotópica tecidual refletirá a assinatura isotópica de uma dieta em particular dependerá, em parte, da taxa de turnover no tecido, pois depende das taxas de aquisição, síntese e catabolismo de cada componente tecidual. Tecidos com turnover rápido refletirão dietas mais recentes, enquanto aqueles com taxas de turnover mais lento refletirão dietas de períodos mais remotos. De modo geral, os tecidos ativos metabolicamente (fígado, pâncreas e tecido gorduroso) apresentam taxas de turnover mais rápidas que os tecidos menos ativos metabolicamente, como o colágeno dos ossos.

Herzka et al. (2001), trabalhando com larvas de uma espécie de pescada (Scianops ocellatus), obtiveram taxas de turnover de carbono e nitrogênio similares. Esses resultados indicam que o destino fisiológico desses elementos deve ser semelhante. Como a maior parte do nitrogênio está contida nas proteínas, o turnover desse elemento deveria refletir mais propriamente o turnover protéico. Contudo, os resultados relatados por esses autores indicam que tanto o turnover do nitrogênio como o de carbono podem ser usados para inferir o turnover protéico, uma vez que os aminoácidos são formados por um esqueleto de carbono, além do grupamento amino; e quase todo carbono apresenta-se contido em proteínas, pois na fase de desenvolvimento inicial o conteúdo de lipídeos corporais é muito reduzido. Dessa forma,

(ㄷ) 2007 Sociedade Brasileira de Zootecnia 
o uso da variação natural da abundância isotópica de carbono apresenta-se como alternativa às metodologias empregadas para avaliar turnover de proteínas teciduais ou corporais.

Hesslein et al. (1993) estudaram mudanças na composição isotópica do enxofre, carbono e nitrogênio em tecidos de Coregonus nasus, em resposta a mudanças na composição isotópica da dieta. Os autores aplicaram um modelo exponencial em função do tempo que discrimina as taxas de substituição do carbono tecidual, decorrentes do acréscimo de tecido (crescimento) e do turnover metabólico. Os mesmos concluíram que para os três isótopos, a maior parte da mudança pode ser atribuída ao crescimento, sendo que a substituição metabólica expressa em turnover foi de apenas 0,1 a $0,2 \%$ dia $^{-1}$.

Fry \& Arnold (1982), utilizando variações naturais na razão isotópica do carbono $\left({ }^{13} \mathrm{C} /{ }^{12} \mathrm{C}\right)$, determinaram turnover desse elemento em póslarva do camarão Penaeus aztecus em cinco experimentos de crescimento. Os autores observaram que o turnover, durante o crescimento acelerado, ocorreu principalmente em função do ganho de peso, apesar do turnover em tecidos animais adultos geralmente ocorrer durante o metabolismo de mantença e ser função do tempo. Assim, no referido trabalho, foi proposto um modelo para avaliar as mudanças de $\mathrm{d}^{13} \mathrm{C}$ em função do incremento de massa corporal.

$\mathrm{Na}$ determinação do turnover tecidual, a utilização da variação natural dos isótopos estáveis do carbono (e/ou nitrogênio) pode ser considerada mais apropriada para avaliar a substituição do carbono tecidual (e/ou nitrogênio) durante períodos mais prolongados (vários dias a alguns meses), fornecendo diretamente o turnover (k), em vez de taxas de síntese e de degradação separadamente. Nesse contexto, torna-se mais adequado considerar as etapas de criação (larvicultura, alevinagem, crescimento etc.). Além disso, os isótopos estáveis possibilitam integrar as variações naturais do ciclo diurno de utilização da proteína em animais que se alimentam em refeições definidas, bem como incorporar as oscilações no desenvolvimento (crescimento multifásico), como as demonstradas para vários animais por Koops \& Grossman (1991) e Tornero (1996).

Gannes et al. (1998), em uma revisão sobre o uso potencial da variação natural dos isótopos

๑ 2007 Sociedade Brasileira de Zootecnia estáveis, destacam o papel dos fisiologistas no uso dessa ferramenta para esclarecer questões fisiológicas e ecológicas, bem como assegurar os fundamentos teóricos e experimentais para aplicação dos isótopos estáveis em outras áreas. Dentre as diretrizes para futuras pesquisas com isótopos estáveis destacadas por esses autores, receberam ênfase o balanço protéico, a alocação de nutrientes e o turnover tecidual.

Zuanon (2003), avaliando o turnover do carbono muscular de tilápias do Nilo em crescimento, verificou que o modelo matemático proposto para avaliar a substituição do carbono tecidual em animais adultos mostrou-se adequado para animais em desenvolvimento. De acordo com o autor, o acréscimo de massa tecidual decorrente do crescimento é o principal fator na determinação da velocidade de diluição isotópica do carbono tecidual, em relação ao turnover metabólico, em animais jovens.

\section{Diluição isotópica}

Quando duas fontes, que diferem isotopicamente entre si, são misturadas uniformemente, a composição do produto gerado reflete a contribuição isotópica destas duas fontes, assim como, a quantidade de cada uma delas. Este é o princípio básico da diluição isotópica.

Dada duas fontes (A') e (B') precursoras de um produto $(\mathrm{P})$, o balanço de massa exprime-se por:

$$
\mathbf{A}^{\prime}+\mathbf{B}^{\prime}=\mathbf{P}
$$

Com o conhecimento da concentração isotópica (enriquecimento relativo) dos ingredientes e do produto, o balanço de massa e isotópico é expresso por:

$$
\delta A^{\prime}\left(A^{\prime}\right)+\delta B\left(B^{\prime}\right)=\delta P(P)
$$

Normalmente necessita saber o percentual das fontes participativas no produto. Assim procedendo, divide-se pela massa do produto $(\mathrm{P})$ :

$$
\begin{aligned}
& \delta A^{\prime}\left(A^{\prime} / P\right)+\delta B^{\prime}\left(B^{\prime} / P\right)=\delta P \\
& \delta A(A)+\delta B(B)=\delta P
\end{aligned}
$$

Como existem somente duas fontes $\mathrm{A}+\mathrm{B}=1$, pode-se exprimir a contribuição relativa de quaisquer fontes no produto. 
O modelo de mistura isotópica só é válido para duas fontes e um produto quando se utiliza isótopos estáveis de um único elemento químico, como por exemplo, o ${ }^{15} \mathrm{~N}$ ou ${ }^{13} \mathrm{C}$, expresso na terminologia delta per mil $\left(\delta^{15} \mathrm{~N}\right.$ ou $\left.\delta^{13} \mathrm{C}\right)$ ou em unidades de átomos $\%{ }^{13} \mathrm{C}$ ou átomos $\%{ }^{15} \mathrm{~N}$.

Dependendo da complexidade da formação do produto, pode-se utilizar sistema de equações com duas fontes $\left(\mathrm{F}_{1}\right.$ e $\left.\mathrm{F}_{2}\right)$ e dois isótopos, como por exemplo:

$$
\begin{array}{ll}
\text { Produto } & =\text { Fonte } \mathrm{F}_{1}+\text { Fonte } \mathrm{F}_{2} \\
\delta^{13} \mathrm{C} & =\mathrm{a} \delta \mathrm{F}_{1}+\mathrm{b} \delta \mathrm{F}_{2} \\
\delta^{15} \mathrm{~N} & =\mathrm{a} \delta \mathrm{F}_{1}+\mathrm{b} \delta \mathrm{F}_{2}
\end{array}
$$

ou, com três fontes participativas $\left(\mathrm{F}_{1}, \mathrm{~F}_{2}, \mathrm{~F}_{3}\right)$ na formação do produto $(\mathrm{P})$.

$$
\begin{aligned}
\text { Produto } & =\text { Fonte } \mathbf{F}_{1}+\text { Fonte } \mathbf{F}_{2}+\text { Fonte } \mathbf{F}_{3} \\
\delta^{13} \mathbf{C} & =\mathbf{a} \delta \mathbf{F}_{1}+\mathbf{b} \delta \mathbf{F}_{2}+\mathbf{c} \delta \mathbf{F}_{3} \\
\boldsymbol{\delta}^{15} \mathbf{N} & =\mathbf{a} \boldsymbol{\delta}^{\prime} \mathbf{F}_{1}+\mathbf{b} \boldsymbol{\delta}^{\prime} \mathbf{F}_{2}+\mathbf{c} \boldsymbol{\delta}^{\prime} \mathbf{F}_{3}
\end{aligned}
$$

para as quais os pesos $(a+b+c=1)$ são os mesmos nas duas equações do balanço de massa e isotópico.

Dietas de diferentes origens, por exemplo, vegetal e animal, que compõem a alimentação de um animal, podem apresentar fatores de fracionamento diferentes, além de apresentarem em sua composição relações Carbono/Nitrogênio diferentes. Estes ruídos isotópicos influenciam os valores dos participativos no produto.

No sentido de evitar o fator de fracionamento entre a fonte e o produto ou mesmo as diferentes concentrações de $\mathrm{C} / \mathrm{N}$ entre diversas fontes e o produto, a experiência tem recomendado a análise de amostras considerando, por exemplo: Fonte proteína e Produto proteína ou Fonte fezes e Produto fezes.

Entretanto, o sistema de equações é limitado pelo número de isótopos utilizado (três fontes e dois isótopos diferentes; seis fontes e cinco isótopos diferentes, CHON'S), uma vez que, na natureza, várias fontes participam na manutenção do organismo.

Este impasse foi resolvido por Phillips \& Greeg (2003), que elaboraram um programa computacional de várias fontes com um produto e dois isótopos estáveis, disponível no programa ISSO SOURCE, acessando: http://www.epa.gov/wed/ pages/models.htm.
Recentemente, Hall-Aspland et al.(2005) propôs um modelo de matriz inversa, com um isótopo $\left(\delta^{15} \mathrm{~N}\right)$ e várias fontes, como modelo alternativo.

\section{Potencial de aplicabilidade na aquiicultura}

Variação Natural dos isótopos estáveis do carbono $\left(\delta^{13} C\right)$ na avaliação da preferência alimentar do caranguejo de mangue Sesarma rectum Randall, 1840 em condições de laboratório.

\section{Cecília Margarita Guerrero- OCampo}

(Tese de Doutorado - 2002/UNESP).

\section{Preferência alimentar}

O resultado da análise isotópica do teste de preferência alimentar entre $S$. alterniflora e $R$. mangle é apresentado na Figura 3. Os valores de $\delta^{13} \mathrm{C}$ das fezes dos caranguejos alimentados com dieta mista se aproximou dos valores das fezes dos caranguejos alimentado apenas com $S$. alterniflora indicando maior consumo deste alimento, que correspondeu a 78,2 $\pm 6,7 \%$ (D.P.) com mínimo de $65,0 \%$ e máximo de $85,1 \%$. Enquanto que a proporção consumida de $R$. mangle foi de $21,8 \pm$ $6,7 \%$ com mínimo de $14,9 \%$ e máximo de $35 \%$.

$\mathrm{O}$ resultado da análise isotópica do teste de preferência alimentar entre $S$. alterniflora e $H$. tiliaceus é apresentado na Figura 4. Os valores $\delta^{13} \mathrm{C}$ das fezes dos caranguejos alimentados apenas com S. alterniflora indicando maior pro-porção deste alimento na dieta, que correspondeu à $89,9 \pm 7,8 \%$ (D.P.) com mínimo de $80,7 \%$ e máximo de $100,0 \%$. Enquanto que a proporção consumida de H. tiliaceus foi de $10,1 \pm 7,8 \%$ com mínimo de 0,0\% e máximo de $19,3 \%$.

A composição isotópica da S. alterniflora é típica de planta $\mathrm{C}_{4}$, enquanto que as composições isotópicas de $R$. mangle e $H$. tiliaceus são típicas de plantas $\mathrm{C}_{3}$. As folhas de $S$. alterniflora apresentam baixa concentração de polifenóis e intermediária de nitrogênio com altas concentrações de materiais de difícil digestão como

두 2007 Sociedade Brasileira de Zootecnia 
celulose, hemicelulose e lignina. As folhas de $R$. mangle apresentam maior concentração de nitrogênio como também de polifenóis, mas os valores de hemicelulose, lignina e celulose são baixos. As folhas de H. tiliaceus apresentam o menor conteúdo de nitrogênio e concentração intermediária de polifenóis; o teor de celulose é mais alto entre as plantas analisadas.

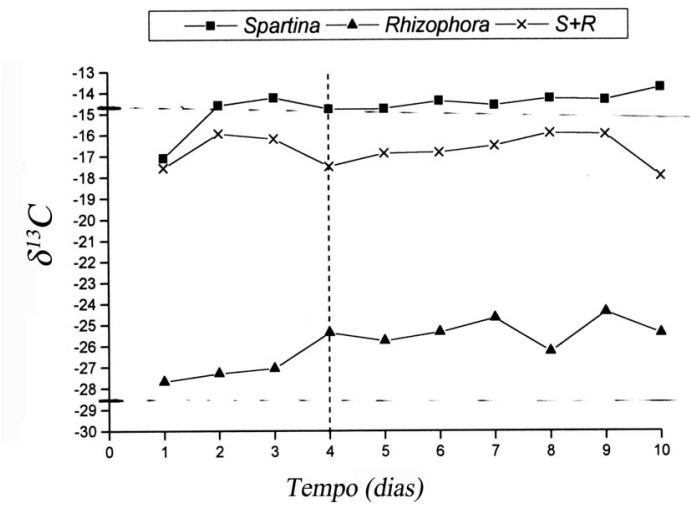

Figura 3 - Sesarma rectum Randall, $1840 . \delta^{13} \mathrm{C}$ das fezes do caranguejo $S$. rectum ao longo do tempo alimentado exclusivamente de $S$. alterniflora, exclusivamente de Rhizophora mangle e de $S$. alterniflora mais $R$. mangle $(S+R)$.

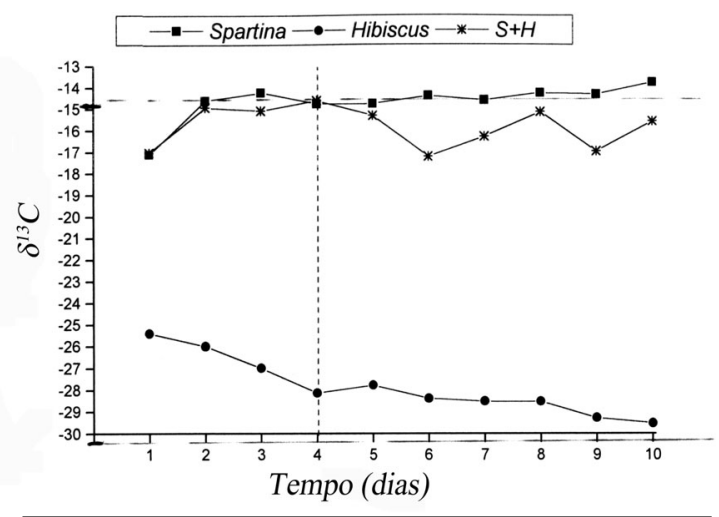

Figura 4 - Sesarma rectum Randall, $1840 . \delta^{13} \mathrm{C}$ das fezes do caranguejo $S$. rectum ao longo do tempo alimentado exclusivamente de $S$. alterniflora, exclusivamente de $H$. tiliaceus e uma mistura de $S$. alterniflora mais $H$. tiliaceus $(\mathrm{S}+\mathrm{H})$.

Além de considerar a celulose, hemicelulose e lignina, os polifenóis são compostos que, geralmente, têm influência na preferência alimentar de herbívoros. Os polifenóis são compostos orgânicos resultantes do metabolismo secundário das plantas, sendo constituídos basicamente de antocianinas, taninos, flavonóides e metilpropanos e atuam ๑) 2007 Sociedade Brasileira de Zootecnia como defensores químicos contra a herbivoria. Estes compostos se ligam às proteínas diminuindo a digestibilidade dos alimentos e possuem propriedades adstringentes que reduzem a palatabilidade das plantas. Os polifenóis parecem que tiveram papel determinante na preferência alimentar de $S$. rectum pelas folhas testadas no experimento. $S$. alterniflora, que possui a menor concentração de polifenóis $\left(2,4 \mathrm{UDO}_{\mathrm{g}} \mathrm{g}^{-1}\right)$, foi a planta mais consumida, enquanto que $R$. mangle e H. tiliaceus, que possuem maiores concentrações (18,6 UDO. $\mathrm{g}^{-1}$ e 10,0 UDO.g ${ }^{-1}$, respectivamente), foram menos consumidas.

\section{$A \delta^{13} \mathrm{C}$ and $\delta^{15} \mathrm{~N}$ tracer study of nutrition in Aquiculture: Penaeus vannamei in a pound growout system}

\section{P.L. Parker, R.K. Anderson, and A. Lawrence}

Stable Isotopes in Ecological. Research. SpringerVerlag. New York. 1989

\section{Teoria e resultados}

A procura de uma dieta adequada para a criação do camarão em tanques de terra é complicada; e é um ótimo exemplo de solução pela técnica da diluição isotópica. Durante a criação, o camarão não só alimenta da ração como também da biota primária do fundo do tanque. Como a análise da excreta ou do tubo digestivo é complexa devido as suas diminutas dimensões, optou-se então pela análise do $\delta^{13} \mathrm{C}$ no músculo do camarão sem o tubo digestivo.

O modelo empregado parte da premissa que o animal é um simples misturador de alimento (biota+ração) necessário ao seu crescimento. Utilizado inicialmente em Penaeus vannamei (camarão), o modelo pode ser estendido a outros animais de pequeno porte que utilizam estas duas fontes, difíceis de serem individualizadas.

Como fontes de alimento foram consideradas a ração $\left(\mathrm{d}_{3}\right)$ e a biota primária $\left(\mathrm{d}_{4}\right)$, de modo que $\mathrm{d}_{3}+\mathrm{d}_{4}=\mathrm{d}$.

O balanço de massa e isotópico expressa-se por:

ração + biota $=$ músculo $($ crescimento $)$

$$
\left(d_{3}\right) \delta d_{3}+\left(d_{4}\right) \delta d_{4}=(d) \delta d
$$


Pela qual, pode-se mensurar o percentual de participação da ração no crescimento, ou que:

$\frac{\mathbf{d}_{3}}{\mathbf{d}}=\frac{\delta(\text { cresciment o })-\delta(\text { biota })}{\delta(\text { ração })-\delta(\text { biota })}$

Pelo mesmo raciocínio, pode-se mensurar o percentual de participação da biota no crescimento do músculo do camarão.

$$
\frac{\mathbf{d}_{4}}{\mathbf{d}}=\frac{\delta(\text { ração })-\delta(\text { crescimento })}{\delta(\text { ração })-\delta(\text { biota })}
$$

A técnica do $\delta^{13} \mathrm{C}$ permite mensurar a contribuição relativa da produtividade primária (biota ou biofilme) versus dieta no crescimento dos juvenis de camarão. A mensuração do $\delta^{13} \mathrm{C}$ no músculo indica o carbono integrado e assimilado durante o tempo de vida, e uma das vantagens da técnica é o fato de que ela registra a matéria orgânica assimilada, em vez da ingerida, e é quantitativa.

Os resultados da alimentação ${ }^{\circ} 4$, das 8 rações testadas, foram:

$\begin{array}{ll}\text { camarão inicial; } & \delta_{\mathrm{i}}=-13,4 \% \text { o } \\ \text { ração; } & \left(\delta_{\mathrm{f}}\right)=-20,3 \% \text { o } \\ \text { a) engorda, terra; } & \left(\delta_{\mathrm{g}}\right)=-16,7 \% \text { o } \\ \text { b) biota inicial, terra; } & \left(\delta_{\mathrm{p}}\right)=-13,1 \% \text { o } \\ \text { c) biota final, terra; } & \left(\delta_{\mathrm{p}}\right)=-15,5 \% \text { o }\end{array}$

\section{Contribuição em peso proveniente} da biota na engorda do camarão

$$
\frac{\text { biota }}{\text { engorda }} ; \frac{d_{4}}{d}=\frac{(-20,3 \%)-(-16,7 \%)}{(-20,3 \%)-(-15,5 \%)}=0,75
$$

\section{Contribuição em peso proveniente da} alimentação (ração) na engorda do camarão

$$
\frac{\text { ração }}{\text { engorda }} ; \frac{d_{3}}{d}=\frac{(-16,7 \%)-(-15,5 \%)}{(-20,3 \%)-(-15,5 \%)}=0,25
$$

$\mathrm{O}$ aspecto mais importante deste trabalho foi a identificação quantitativa das duas fontes de alimentares (biota e dieta) simultaneamente, durante o crescimento ou a engorda do camarão.

Analisando todas as dietas, os resultados indicam que a biota natural, desenvolvida no lago durante a alimentação, contribui na faixa de 44 a $86 \%$ do carbono assimilado pelo camarão durante o seu crescimento. Por diferença, a participação do carbono proveniente da dieta encontra-se na faixa de 56 a $14 \%$ do carbono assimilado.

\section{Crescimento e turnover no processo de diluição isotópica do carbono muscular em tilápia do Nilo (Oreochromis niloticus) por meio dos isótopos estáveis $\left({ }^{13} \mathrm{C} /{ }^{12} \mathrm{C}\right)$}

\section{Jener Alexandre Sampaio Zuanon}

(Tese de Doutorado - 2003/UNESP)

Com o intuito de avaliar a contribuição do crescimento e do turnover para o processo de diluição isotópica no tecido muscular, foram testados modelos matemáticos capazes de predizer as alterações na composição isotópica de carbono muscular, tanto em função do tempo, como do acréscimo de massa corporal. Dois grupos de tilápia do Nilo (Oreochromis niloticus) foram distribuídos em 20 aquários cada (250L), em sistema de recirculação de água dotado de filtragem e manutenção da temperatura a $25 \pm$ $0,5^{\circ} \mathrm{C}$. Os peixes foram alimentados com dietas isoprotéicas (32\% PD) e isocalóricas (3200 kcal/ $\mathrm{kg}$ ED) à base de plantas dos ciclos fotossintéticos $\mathrm{C}_{3}$ ou $\mathrm{C}_{4}$, para certificar que cada grupo apresentasse uniformidade na composição isotópica do carbono muscular. Após o estabelecimento do patamar de equilíbrio isotópico, as dietas foram trocadas entre os grupos de peixes (grupo 1: $\mathrm{C}_{3} \rightarrow$ $\mathrm{C}_{4}$; grupo $2: \mathrm{C}_{4} \rightarrow \mathrm{C}_{3}$ ) até estabelecer um novo patamar de equilíbrio isotópico de carbono. Para as análises isotópicas, foram coletadas aleatoriamente três amostras de tecido muscular de cada tratamento, ao longo do período experimental.

Não foram observadas diferenças significativas para os parâmetros de desempenho entre os tratamentos. Foram obtidos bons ajustes dos modelos que avaliam a variação de $\delta^{13} \mathrm{C}$ em função do tempo, resultando em valores para a meia vida do carbono de 23,33 dias para o grupo $\mathrm{C}_{3} \rightarrow \mathrm{C}_{4} \mathrm{e}$ 25,96 dias para o grupo $\mathrm{C}_{4} \rightarrow \mathrm{C}_{3}$. Com base nos resultados das constantes de diluição isotópicas

두 2007 Sociedade Brasileira de Zootecnia 
do carbono muscular proveniente do crescimento $\left(0,0263 \mathrm{dia}^{-1}\right.$ e $\left.0,0254 \mathrm{dia}^{-1}\right)$ e de turnover $(0,0034$ $\mathrm{dia}^{-1}$ e $0,0013 \mathrm{dia}^{-1}$ ) pode-se concluir que a maior parte da diluição isotópica pode ser atribuída ao crescimento. A aplicação de modelos que avaliam a variação de $\delta^{13} \mathrm{C}$ em função do acréscimo de massa corporal não proporcionou resultados que tenham explicação biológica aparente.

\section{Organismos vivos e dietas secas na larvicultura do pacu Piaractus mesopotamicus e o uso dos isótopos estáveis de carbono $\left(\delta^{13} \mathrm{C}\right)$ e nitrogênio $\left(\delta^{15} \mathrm{~N}\right)$ como indicadores naturais da incorporação do alimento no tecido larval}

\section{Rosângela Kiyoko Jomori}

(Tese de Doutorado - 2005/UNESP).

Larvas de pacu $P$. mesopotamicus, com cinco dias de vida e iniciando a alimentação exógena, foram alimentadas exclusivamente com náuplios de Artemia (A) ou dieta seca (D); receberam alimentação mista por todo o período $(\mathrm{A}+\mathrm{D})$; ou tiveram os náuplios substituídos por dieta seca após três (A3D), seis (A6D) e 12 dias (A12D) do início da alimentação.

Logo após o fornecimento do alimento exógeno, as larvas começaram a apresentar os sinais da incorporação dos nutrientes dos alimentos. A composição isotópica inicial da larva (antes de receber os alimentos) que era de $-20,2 \%$ o $\left(\delta^{13} \mathrm{C}\right)$ e $9,5 \%$ ( $\left(\delta^{15} \mathrm{~N}\right)$ alterou para $-16,8 \%$ e $7,9 \%$, respectivamente, em três dias de alimentação com náuplios de Artemia, seguindo em direção ao sinal do alimento $\left(\delta^{13} \mathrm{C}_{\text {náuplios }}=-15,1 \%\right.$ e $\delta^{15} \mathrm{~N}_{\text {náuplios }}$ $=4,6 \%$ ). Gradativamente, as mudanças isotópicas continuaram em relação à composição inicial das larvas e, com o decorrer do tempo, os animais alimentados com náuplios de Artemia (Larvas A) estabeleceram um patamar de equilíbrio isotópico (valor constante), já por volta de 24 dias de alimentação, num valor mais rico que o alimento da ordem de $-12,6 \%$ para o $\delta^{13} \mathrm{C}$ e $7,0 \%$ o para o $\delta^{15} \mathrm{~N}$. As larvas alimentadas exclusivamente com a dieta seca (Larvas D) também alteraram sua composição isotópica em relação ao valor inicial, mas as mudanças ocorreram mais lentamente, de maneira que esses animais não atingiram o patamar de equilíbrio isotópico num período de 42 dias de alimentação. Essas diferenças podem ser explicadas pelas repostas de crescimento. Até 36 dias de alimentação, na média, o crescimento em peso das Larvas A foi superior em cerca de 5 vezes em relação às Larvas $\mathrm{D}$.

Nas larvas que passaram pelo processo da substituição do alimento vivo, mais precoce (A3D e A6D) ou tardiamente (A12D), e naquelas que receberam os dois alimentos por todo o período $(\mathrm{A}+\mathrm{D})$, claramente, puderam ser observados diferentes momentos em que os animais começaram a apresentar sinais da dieta seca em seus tecidos, indicando diferenças no comportamento alimentar desses animais frente à disponibilidade do alimento seco.

Nas Larvas A3D e A6D, enquanto o alimento eram os náuplios de Artemia, os valores isotópicos foram semelhantes aos das larvas que receberam somente alimento vivo (Larvas A). Após a supressão dos náuplios de Artemia, verificou-se que a composição isotópica dos animais A3D e A6D começou a se diferenciar da composição das Larvas A. Os sinais isotópicos das Larvas A e A3D que eram similares aos três dias de alimentação $\left(\sim \delta^{13} \mathrm{C}=-16,8 \%\right.$ ) se diferenciaram aos seis dias, quando as Larvas A3D já não recebiam mais o alimento vivo. As Larvas A continuaram traçando um perfil isotópico em direção ao sinal do alimento vivo (mais rico em ${ }^{13} \mathrm{C}$ ) e apresentaram uma mudança de $-16,8 \%$ o para $-14,8 \%$ aos seis dias; já as Larvas A3D, nesse mesmo período, começaram a ficar mais pobre em ${ }^{13} \mathrm{C}$ (por causa da dieta seca) alterando a composição de $-16,8 \%$ o para $-17,1 \%$. A partir daí, Larvas A3D desenharam, ao longo do tempo, um traçado em direção ao sinal das larvas que haviam recebido apenas dieta seca $\left(\delta^{13} C_{\text {dieta seca }}=-25,0 \%\right)$. Esse comportamento também foi observado para os valores de $\delta^{15} \mathrm{~N}$, assim como no tratamento A6D, tão $\operatorname{logo}$ a substituição dos náuplios por dieta seca.

Os resultados de crescimento em comprimento e peso dos animais nos tratamentos A3D e A6D não demonstraram crescimento expressivo proporcionado pela dieta seca logo após a transição alimentar, o que não permitiu fazer inferência sobre o aproveitamento da dieta seca nessa fase. Por outro lado, a sensibilidade da metodologia dos 
isótopos estáveis permitiu a constatação de que acréscimos mínimos de massa nos tecidos provocaram alterações dos sinais isotópicos desses animais.

A substituição total dos náuplios a partir de 12 dias de alimentação não prejudicou o crescimento dos animais e nem a sobrevivência, diferentemente do verificado nas substituições mais precoces (A3D e A6D). Pela análise dos isótopos estáveis, a composição das Larvas A12D que progressivamente se tornava mais rica em ${ }^{13} \mathrm{C}$ até cerca de 12 dias: $-15,4 \%$; $-14,4 \%$ e $-13,9 \%$ aos seis, nove e 12 dias, respectivamente (por causa dos valores dos náuplios: $\delta^{13} \mathrm{C}=-15,1 \%$ ), alterou para $-18,2 \%$ aos 18 dias, logo após a supressão total do alimento vivo $\left(\delta^{13} C_{\text {dieta seca }}=-25,0 \%\right.$ ). Nas análises posteriores, o traçado isotópico desenhado foi a de um perfil em direção ao sinal das Larvas $\mathrm{D}$, ficando claramente evidenciado o potencial de aproveitamento da dieta seca pelas larvas nesse estágio do desenvolvimento, animais com cerca de $10 \mathrm{mg}$ de peso úmido.

$\mathrm{O}$ início da busca natural pela dieta seca nas Larvas A+D foi constatado entre 12 a 18 dias de alimentação. Esses animais começaram a ficar mais leve em ${ }^{13} \mathrm{C}$ por causa da dieta seca $\left(\delta^{13} \mathrm{C}_{\text {dieta }}\right.$ seca $=-25,0 \%$ o); entretanto, Larvas A12D, que tiveram o alimento vivo totalmente suprimido a partir de 12 dias, alteraram mais rapidamente sua composição isotópica a partir deste período. Em $\delta^{13} \mathrm{C}$ essa mudança foi de $-13,9 \%$ aos 12 dias para $-20,6 \%$ aos 24 dias; enquanto Larvas A+D apresentaram uma mudança progressivamente mais lenta, de $-13,6 \%$ aos 12 dias para $-14,1 \%$ o aos 24 dias. Isso revelou que os animais $A+D$ continuaram utilizando o alimento vivo que estava ainda sendo oferecido, mesmo sendo capazes de aproveitar eficientemente a dieta seca já neste período. Por outro lado, a busca voluntária da dieta seca ao longo do tempo no tratamento $\mathrm{A}+\mathrm{D}$ tornouse cada vez mais evidente, pois as Larvas $A+D$ abandonaram totalmente o perfil isotópico das Larvas A e foram ao encontro daquelas alimentadas exclusivamente com dieta seca (Larvas D). Após 42 dias de período experimental, os animais apresentavam uma composição em $\delta^{13} \mathrm{C}$ da ordem de: $-12,6 \%$ (Larvas A); -23,4\%o (Larvas A+D) e $22,7 \%$ (Larvas D). $\left(\delta^{13} \mathrm{C}_{\text {náuplios }}=-15,1 \% \circ ; \delta^{13} \mathrm{C}_{\text {dieta }}\right.$ seca $=-25,0 \%$ ).

Os isótopos estáveis de carbono e nitrogênio foram eficientes como indicadores naturais dos alimentos aproveitados pelos animais na fase larval. Para as pesquisas que abordam aspectos sobre a alimentação e nutrição de larvas de peixes, além das informações científicas sobre a assimilação dos nutrientes das dietas ao longo do desenvolvimento larval, uma grande contribuição do estudo foi mostrar que os isótopos estáveis, seguindo as premissas metodológicas necessárias, é uma ferramenta que pode ser utilizada nesse campo pela sensibilidade e precisão do método, através da retenção dos átomos de carbono e nitrogênio dos alimentos no tecido animal.

\section{Literatura citada}

DUCATTI, C.; CARRIJO, A.S.; PEZZATO, A.C. et al. Modelo teórico e experimental da reciclagem do carbono-13 em tecidos de mamíferos e aves. Scientia Agrícola, v.59, n.1, p.29-33, 2002.

DUCATTI, C. Isótopos Estáveis Ambientais. Botucatu. Universidade Estadual Paulista, 2006. 204 p. Apostila.

FRY, B.; ARNOLD, C. Rapid ${ }^{13} \mathrm{C} /{ }^{12} \mathrm{C}$ turnover during growth of brow shrimp (Penaeus aztecus). Oecologia, Barcelona, v.54, p.200-204, 1982.

GANNES, L.Z.; DEL-RIO, C.M.; KOCH, P. Natural abundance variations in stable isotopes and their potential uses in animal physiology ecology. Comp. Biochem. Physiol., 199A, v.3, p.725-737, 1998

GUERRERO-OCAMPO, C.M. Hábito alimentar em caranguejos grapsídeos de manguezais. Tese (Doutorado em Zoologia) - Instituto de Biociências, Universidade Estadual Paulista, Botucatu. 2002. 148p.

HALL-ASPLAND, S.A.; HALL, A.P.; ROGERS, T.L. A new approch to the solution of the linear mixing model for a single isotope: application to the case of an oppotunistic predator. Oecologia, v.143, p.143-147, 2005.

HERKZA, S.Z.; HOLT, S.A.; HOLT, G.J. Documenting the settlement history of individual fish larvae using stable isotope ratios: model development and validation. Journal of Experimental Marine Biology and Ecology, v.265, p.4974, 2001.

HESSLEIN, R.H.; HALLARD, K.A.; RAMLAL, P. Replacement of súlfur, carbon, and nitrogen in tissue of growing broad whitefish (Coregonus nasus) in response to a change in diet traced by $\delta^{34} \mathrm{~S}, \delta^{13} \mathrm{C}, \delta^{15} \mathrm{~N}$. Canadian of Journal Fisheries and Aquatic Science, v.50, p.2071-2076, 1993.

JOMORI, R.K. Organismos vivos e dietas secas na larvicultura do pacu Piaractus mesopotamicus e o uso dos isótopos estáveis de carbono $\left(\delta^{13} \mathrm{C}\right)$ e nitrogênio $\left(\delta^{15} \mathrm{~N}\right)$ como indicadores naturais da incorporação do alimento no tecido larval. Tese (Doutorado em Aqüicultura) - Centro de Aqüicultura, Universidade Estadual Paulista, Jaboticabal. 121p., 2005.

KENNEDY, B.V.; KROUSE, H.R. Isotope fractionation by plants and animals: implications for nutrition research. Canadian Journal Physiology and Pharmacology, v.68, p.960-972, 1990.

KOOPS, W.J.; GROSSMAN, M. Multifasic growth and allometry. Growth Dev. Aging 55, p. 203-212, 1991.

PARKER, P.L.; ANDERSON, R.K.; LAWRENCE, A. A $\delta^{3} \mathrm{C}$ and $\delta^{15} \mathrm{~N}$ tracer study of nutrition in aquiculture: Penaeus vannamei in a pond growout system. In: RUNDEL P.W.;

() 2007 Sociedade Brasileira de Zootecnia 
EHLERINGER, J.R.; NAGY, K.A. (Ed). Stable isotopes in ecological research. Springer-Verlag, New York. Vol. 68. Section II, 1989. P.288-303.

PHILLIPS, D.L.; GREEG, J.W. Source partitioning using stable isotopes: coping with too many sources. Oecologia, v.136, p.261-269, 2003.

TORNERO, M.T.T. Utilização de modelos assintóticosigmóides de crescimento multifásico com sazonalidade em estudos zootécnico. Tese (Doutorado), Faculdade de Medicina Veterinária e Zootecnia - UNESP, Botucatu, 131pp, 1996.

ZUANON, J.A.S. Turnover de carbono em tecido muscular de alevinos de tilápia do Nilo (Oreochromis niloticus) por meio dos isótopos estáveis de carbono $\left({ }^{13} \mathrm{C} /{ }^{12} \mathrm{C}\right)$. Jaboticabal: Centro de Aqüicultura, Universidade Estadual Paulista, 2003. 96p. Tese (Doutorado em Aqüicultura) Centro de Aqüicultura, 2003. 